\title{
Image-Guided Surgery in Patients with Pancreatic Cancer: First Results of a Clinical Trial Using SGM-101, a Novel Carcinoembryonic Antigen-Targeting, Near-Infrared Fluorescent Agent
}

Charlotte E. S. Hoogstins, MD ${ }^{1,2}$, Leonora S. F. Boogerd, MD ${ }^{1}$, Babs G. Sibinga Mulder, BSc ${ }^{1}$, J. Sven D. Mieog, MD, $\mathrm{PhD}^{1}$, Rutger Jan Swijnenburg, MD, $\mathrm{PhD}^{1}$, Cornelis J. H. van de Velde, MD, PhD ${ }^{1}$, Arantza Farina Sarasqueta, MD, $\mathbf{P h D}^{3}$, Bert A. Bonsing, MD, $\mathbf{P h D}^{1}$, Berenice Framery, $\mathrm{MSc}^{4}$, André Pèlegrin, $\mathrm{PhD}^{5}$, Marian Gutowski, $\mathrm{MD}^{6}$, Françoise Cailler, $\mathrm{PhD}^{4}$, Jacobus Burggraaf, $\mathrm{MD}, \mathrm{PhD}^{2,7}$, and Alexander L. Vahrmeijer, MD, $\mathbf{P h D}^{1}$

${ }^{1}$ Department of Surgery, Leiden University Medical Center, Leiden, The Netherlands; ${ }^{2}$ Centre for Human Drug Research, Leiden, The Netherlands; ${ }^{3}$ Department of Pathology, Leiden University Medical Center, Leiden, The Netherlands; ${ }^{4}$ Surgimab, Montpellier, France; ${ }^{5}$ Institut de Recherche en Cancérologie de Montpellier, Montpellier, France; ${ }^{6}$ Institut Régional du Cancer de Montpellier, Montpellier, France; ${ }^{7}$ Leiden Academic Center for Drug Research, Leiden, The Netherlands

\begin{abstract}
Background. Near-infrared (NIR) fluorescence is a promising novel imaging technique that can aid in intraoperative demarcation of pancreatic cancer (PDAC) and thus increase radical resection rates. This study investigated SGM-101, a novel, fluorescent-labeled anticarcinoembryonic antigen (CEA) antibody. The phase 1 study aimed to assess the tolerability and feasibility of intraoperative fluorescence tumor imaging using SGM-101 in patients undergoing a surgical exploration for PDAC.

Methods. At least $48 \mathrm{~h}$ before undergoing surgery for PDAC, 12 patients were injected intravenously with $5,7.5$, or $10 \mathrm{mg}$ of SGM-101. Tolerability assessments were performed at regular intervals after dosing. The surgical field was imaged using the Quest NIR imaging system. Concordance between fluorescence and tumor presence on histopathology was studied.
\end{abstract}

Charlotte E. S. Hoogstins and Leonora S. F. Boogerd contributed equally and share first authorship.

(C) The Author(s) 2018

First Received: 6 March 2018; Published Online: 26 July 2018

A. L. Vahrmeijer, MD, PhD

e-mail: a.1.vahrmeijer@lumc.nl
Results. In this study, SGM-101 specifically accumulated in CEA-expressing primary tumors and peritoneal and liver metastases, allowing real-time intraoperative fluorescence imaging. The mean tumor-to-background ratio (TBR) was 1.6 for primary tumors and 1.7 for metastatic lesions. One false-positive lesion was detected (CEA-expressing intraductal papillary mucinous neoplasm). False-negativity was seen twice as a consequence of overlying blood or tissue that blocked the fluorescent signal.

Conclusion. The use of a fluorescent-labeled anti-CEA antibody was safe and feasible for the intraoperative detection of both primary PDAC and metastases. These results warrant further research to determine the impact of this technique on clinical decision making and overall survival.

Surgical resection is the only possible curative treatment for patients with pancreatic ductal adenocarcinoma (PDAC). Differentiation between tumoral and non-tumoral tissue is often difficult during PDAC surgery, and this can lead to incomplete cancer removal, exemplified by high percentages of irradical resections. ${ }^{1-3}$ As survival time doubles for patients with a microscopically radical resection, ${ }^{4}$ enhanced intraoperative visualization of PDAC is crucial to increase radical resection rates and improve 
patient outcomes. Image-guided surgery using near-infrared (NIR) fluorescence is a novel imaging technique that can aid in real-time demarcation of tumors during surgery. ${ }^{5}$

To detect tumors using NIR fluorescence imaging, a fluorescent contrast agent is administered, and the distribution of the agent is visualized using a dedicated imaging system. At first, the fluorescence-imaging field focused predominantly on indocyanine green (ICG), a nonspecific imaging agent, because ICG is available for clinical use. Exploiting the hypothesized passive accumulation of ICG in tumor cells, a study in PDAC was performed. ${ }^{6}$ The results were unfavorable because sufficient contrast between the tumor and surrounding pancreatic tissue was not achieved.

Currently, the fluorescence-imaging field focuses mainly on tumor-specific agents (e.g., antibodies or ligands conjugated to a fluorophore) that molecularly target biomarkers expressed by tumor cells. Recently, clinical trials (including one randomized controlled trial) with these tumor-specific fluorescent agents in other cancer types have demonstrated feasibility and potential clinical benefits of image-guided surgery. ${ }^{7-9}$ However, tumor-specific agents for fluorescence imaging of PDAC have not been investigated.

A number of tumor-specific imaging agent seem to have high potential for specific fluorescence imaging of PDAC including integrin $\alpha \mathrm{v} \beta 6$, carcinoembryonic antigen (CEA), epithelial growth factor receptor (EGFR), and urokinase plasminogen activator receptor (uPAR). ${ }^{10}$ As a glycoprotein produced by gastrointestinal tissue, CEA is overexpressed in many cancer types, including pancreatic ductal adenocarcinoma. ${ }^{11,12}$ Antibodies directed toward CEA-overexpressing tumors for various therapeutic applications have been successfully tested in trials. ${ }^{13-15}$ Moreover, imaging studies using positron emission tomography (PET) and radiolabelled anti-CEA antibodies have demonstrated high tumor uptake and good contrast. ${ }^{16,17}$

This study investigated the use of a novel fluorescentlabeled $(700 \mathrm{~nm})$ anti-CEA antibody for clinical application. In PDAC orthotopic mouse models using BxPC3 cells, different fluorescent-labeled anti-CEA antibodies have allowed clear tumor delineation. ${ }^{18-20}$ Our study aimed to assess the tolerability and feasibility of intraoperative fluorescence tumor imaging using SGM-101 for patients undergoing a surgical exploration for PDAC.

\section{METHODS}

\section{Study Design}

This study was an open-label, single-ascending-dose, exploratory study of 12 patients approved by a certified medical ethics review board (clinicaltrials.gov ID: NCT02973672). The study primarily aimed to assess SGM101 with respect to tolerability and performance in the intraoperative detection of PDAC lesions.

Due to the exploratory nature of the study, sample size was not based on a formal calculation of statistical power. A doseescalating scheme with intravenous doses of 5,7.5 and $10 \mathrm{mg}$ administered 48 or $96 \mathrm{~h}$ before surgery was used based on our preclinical data. ${ }^{18} \mathrm{We}$ included patients with elevated plasma CEA levels $(>3 \mathrm{ng} / \mathrm{mL})$ who had a clinical diagnosis of PDAC and were scheduled for surgical exploration.

The exclusion criteria ruled out pregnancy, history of allergic reactions, impaired renal function, plasma CEA level higher than $300 \mathrm{ng} / \mathrm{mL}$ and a diagnosis of another malignancy within the last 5 years. Tolerability assessment (adverse events, electrocardiogram, blood pressure, pulse, peripheral oxygen saturation, respiratory rate, and temperature), routine laboratory tests, and urine collection for urinalysis were performed at regular intervals, starting just before SGM-101 administration and lasting until $12 \mathrm{~h}$ after dosing.

On both the day of surgery and the first postoperative day, measurements were repeated. Follow-up visits coinciding with clinical care took place on the day of discharge and at the first outpatient clinic visit. Adverse events and the concomitant use of other medications were recorded throughout the study period.

All surgical procedures were open procedures, and the surgical field was explored using standard visual and tactile methods, complemented with ultrasound based on the surgeon's preference. Subsequently, the fluorescence imaging system was used to identify NIR-fluorescent lesions. All lesions identified by visual/tactile methods and/or NIR fluorescence were resected if resection was surgically feasible and clinically relevant. Each resected lesion was marked on a case report form as fluorescent or non-fluorescent and as clinically suspected of malignancy or not.

After resection, fluorescence imaging of the resected specimen (before and after slicing) was performed in the pathology department. The slice containing the peak fluorescence signal was imaged using the Pearl imager and corresponding software (LI-COR, Lincoln, NE, USA). The fluorescence signal in tumor and background tissue (tumorto-background ratio [TBR]) was quantified using ImageJ (version 1.49b; NIH, Bethesda, MD, USA; http://imagej. nih.gov/ij/) on tagged image file format (TIFF) images (8 bits) subtracted from intraoperative videos. Tumor status was correlated with intraoperative fluorescent assessment. A tumor-positive, fluorescent lesion was regarded as a truepositive. A tumor-negative, fluorescent lesion was regarded as a false-positive, and a tumor-positive, non-fluorescent lesion was regarded as a false-negative. 
In addition, immunohistochemistry (IHC) was performed to demonstrate CEA expression. To correlate SGM-101 presence with tumor status and CEA expression on a microscopic level, sections were scanned using the Odyssey imager (LI-COR). In this report, both TBR and patient characteristics are reported as mean \pm standard deviation and range.

\section{Investigational Product}

The tumor-specific imaging agent, SGM-101 (molecular weight, $148.6 \mathrm{kDa}$ ), consists of an anti-CEA chimeric monoclonal antibody covalently bound to fluorophore BM104 (excitation, $686 \mathrm{~nm}$; emission, $704 \mathrm{~nm}$ ). Approximately $30-40 \%$ of the antibody is unconjugated, and the average number of fluorophores per antibody is between 1 and 2 .

In compliance with good manufacturing practice (GMP), SGM-101 was manufactured by Novasep (Gosselies, Belgium). The agent was supplied by Surgimab (Montpellier, France) as a sterile solution for injection in amber glass vials containing $10 \mathrm{mg}$ of SGM-101 in $10 \mathrm{~mL}$ and stored in frozen form at $-20{ }^{\circ} \mathrm{C}$. Before SGM-101 administration, the frozen vials were thawed and diluted in $100 \mathrm{~mL}$ of $0.9 \% \mathrm{NaCl}$ and infused for $30 \mathrm{~min}$.

\section{Imaging System}

Imaging was performed using the Artemis and Spectrum fluorescence imaging systems (Quest Medical Imaging, Middenmeer, the Netherlands ${ }^{21}$ ). Both systems consist of three wavelength-isolated light sources, including a "white" light source and two separate NIR light sources.

For this study, the Cy5,5 filter setting (fluorescent range, $680 \pm 30 \mathrm{~nm}$ ) was used. Color video and fluorescence images were acquired simultaneously by separate sensors and displayed in real time using custom-built optics and software, thereby displaying color video and NIR fluorescence images separately. A pseudo-colored (lime green) merged image of the color video and fluorescence images also was generated. The gain and exposure time settings were controlled using the Quest software. An average gain setting of 25 was used, and exposure time was varied between 60 and $120 \mathrm{~ms}$ according to the clinical situation. The camera was attached to a freely moveable arm. During surgery, the camera and moveable arm were enclosed in a sterile shield and drape (Medical Technique Inc., Tucson, AZ, USA).

\section{RESULTS}

\section{General}

The study investigated 12 patients. Demographics and details on surgical procedure are summarized in Table 1.

\section{Dose Escalation}

Because the increase from 5 to $7.5 \mathrm{mg}$ did not cause an increase in TBR, the interval between SGM-101 administration and surgery was prolonged to $96 \mathrm{~h}$, while the dose remained equal at $7.5 \mathrm{mg}$ for the following cohort. The longer interval between SGM-101 administration and surgery resulted in an improvement of TBR. Hence, the last dose level was increased to $10 \mathrm{mg}$, while the longer interval was retained. The TBR results are summarized in Fig. 1.

\section{Safety Data}

One patient (ID no. 208) experienced mild, self-limiting abdominal discomfort and diarrhea on the day of SGM-101 administration (dose, $7.5 \mathrm{mg}$ ), although these symptoms likely were not related to SGM-101. Causality could not be excluded. In the remaining patients, adverse events related to SGM-101 were not seen. After the surgical procedure, four severe adverse events were noted, all related to the surgical procedure or to disease progression.

\section{Primary Tumor}

In one patient (ID no. 206), the surgical procedure was abandoned before the primary tumor was visualized after detection of occult liver metastases. In the remaining 11 patients, the primary tumor was always visualized with fluorescence imaging (TBR, $1.6 \pm 0.37$; range, 1.3-2.3; Fig. 2 and Table 1). Tumor specimens were not available if the surgical procedure was abandoned due to unresectability or metastases. Consequently, ex vivo fluorescence measurements and correlation of fluorescence signal and histopathology were assessed in seven primary tumors. Assessment of one slice containing peak fluorescence per resection specimen with the Pearl demonstrated a mean TBR of $3.2 \pm 0.79$ (range, 1.7-4.1; Table 1). Six primary tumors were confirmed as adenocarcinomas on histopathology. The IHC for CEA showed moderate to strong CEA overexpression (Table 1). Odyssey scans of the fluorescence signal in the primary tumor sections showed concordance with tumor cells on histopathology (Fig. 2). The remaining tumor was an intraductal papillary mucinous neoplasm (IPMN) with low-grade dysplasia. Because this is a pre-malignant condition, the fluorescence signal was deemed false-positive. Moderate CEA expression was present in $10-50 \%$ of IPMN lesional cells, explaining the fluorescence.

\section{Other Lesions}

In three patients, liver and/or peritoneal metastases were identified (with both methods) during surgery, whereas in 


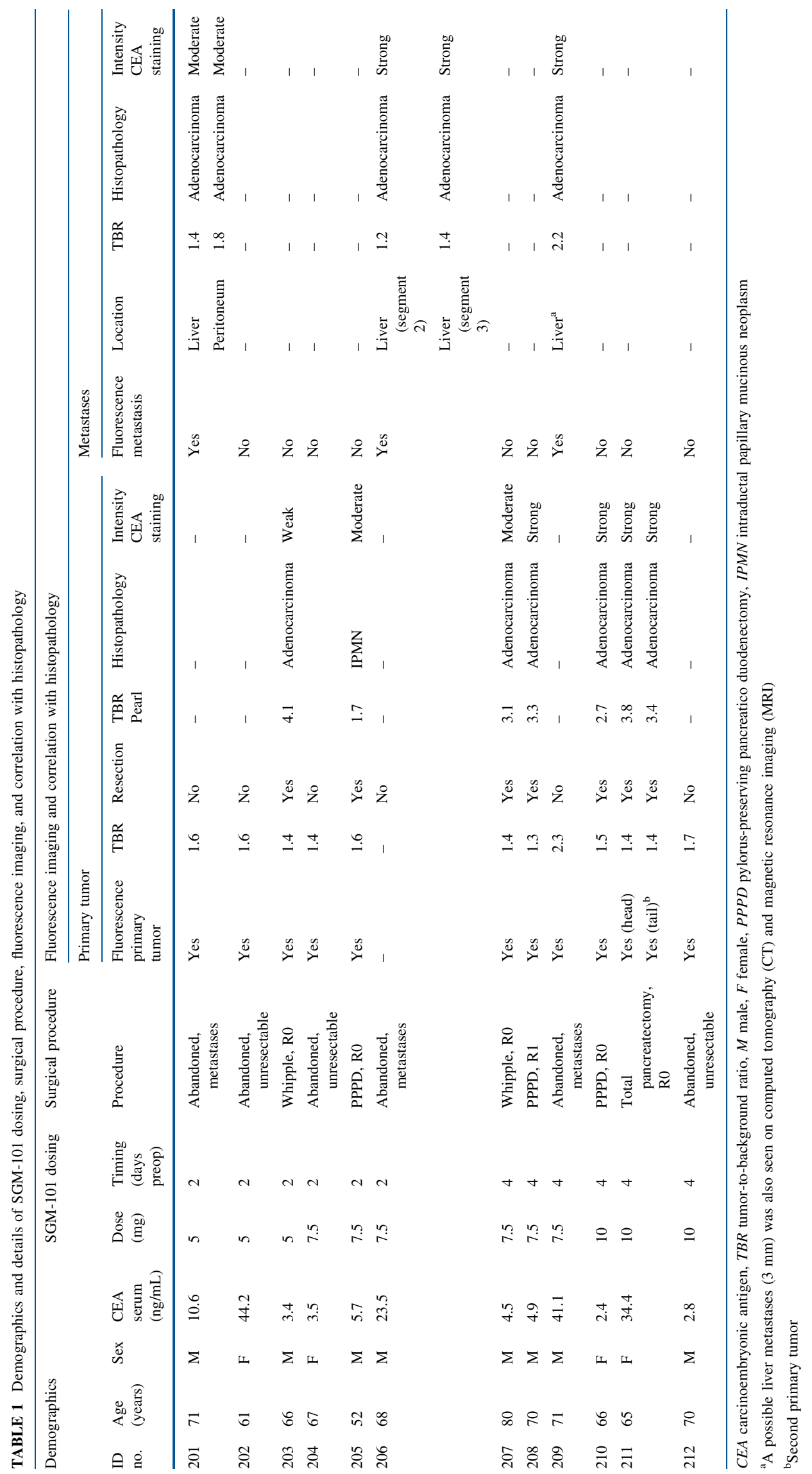




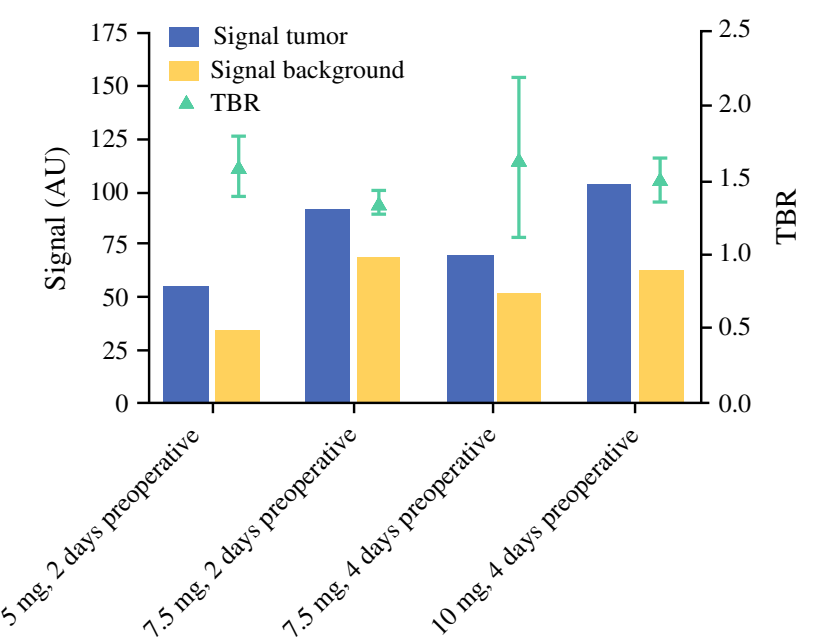

FIG. 1 Tumor and background fluorescence signal (in arbitrary units $[\mathrm{AU}])$ and mean tumor-to-background ratio (TBR) per dose group. The influence of SGM-101 dose and timing on the fluorescence signal and TBR seemed limited in this study

only one patient (ID no. 209), a possible liver metastasis $(3 \mathrm{~mm})$ was seen on cross-sectional imaging (computed tomography $[\mathrm{CT}]$ scan). All metastatic lesions were fluorescent (TBR, $1.7 \pm 0.42$; range, 1.2-2.3; Fig. 3). The IHC for CEA showed moderate to strong CEA overexpression (Table 1). A total of eight clinically suspect, non-fluorescent lesions were resected. In two patients. This nonfluorescent tissue contained malignancy, a suspected tumor ingrowth in the common hepatic artery (TBR, 1.3; ID no. 204), and a suspected 1-cm aorta-caval lymph node (TBR, 1.1; ID no. 207).

In frozen-section analysis, the biopsy of the ingrowth in the common hepatic artery demonstrated adenocarcinoma (i.e., false-negative). Frozen section analysis precluded further ex vivo assessment of this biopsy material. The non-fluorescent lymph node contained a metastasis and also was considered a false-negative. Remarkably, ex vivo imaging using the Pearl demonstrated localization of fluorescence, and IHC staining demonstrated CEA expression.

\section{DISCUSSION}

We assessed the safety and feasibility of intraoperative fluorescence imaging of PDAC using SGM-101 in 12 patients. Administration of SGM-101 doses of 5-10 mg appeared to be safe because the occurrence of a possibly related adverse event was limited to one subject. Moreover, administration of SGM-101 did not cause changes in safety measurements including vital signs, ECG, and routine chemistry, hematology, and coagulation laboratory tests.
Intraoperative fluorescence imaging of PDAC was feasible because fluorescence could be detected in primary tumors as well as liver and peritoneal metastases. This demonstrates that despite the suboptimal intrinsic characteristics of PDAC, including poor vascularization, SGM101 can reach and bind the CEA-expressing tumor cells. However, the TBRs in this study were more modest than for other tumor types (1.6 for primary tumors and 1.7 for metastases). This also could be explained by the histopathology of pancreatic adenocarcinoma, with solitary ducts of tumor cells in preexistent normal pancreatic tissue and a remarkable desmoplastic stroma, which could make the fluorescence pattern more sparse than with other tumor types. Finally, SGM-101 fluoresces at about $700 \mathrm{~nm}$, and this "far red" part of the spectrum is associated with more autofluorescence of the surrounding background.

Overlying tissue and blood in the surgical field likely caused both cases of intraoperative false-negativity in this study. The maximal depth of fluorescence signal penetration is approximately $1 \mathrm{~cm}$ below the tissue surface. ${ }^{22}$ Consequently, more deeply seated tumors will not be detectable using fluorescence imaging. Moreover, absorption of the fluorescence signal by blood can have a negative impact on the detection of the fluorescence signal. ${ }^{23}$ Thus, it may be beneficial to combine fluorescence with other methods, such as radionuclides or photo-acoustic imaging, to increase detection of deeply seated or covered tumors. For example, a study with administration of (111)Ingirentuximab-IRDye800CW has been initiated for patients with clear cell renal cell carcinoma. An ex vivo study with this agent has already demonstrated that uptake in tumor tissue can be visualized using both radionuclide and fluorescence imaging. ${ }^{24}$ The advantage of a radionuclide is not only that it allows increased penetration depth, but also that it also allows preoperative scanning.

Based on both preclinical and clinical studies, CEA was chosen as a suitable target for fluorescence imaging of PDAC. The current study confirmed its potential as a target for intraoperative fluorescence imaging. Because PDAC is characterized by several genetic mutations such as KRAS (90\%), CDK2NA (90\%), TP53 (75-90\%), and SMAD4/ DPDAC4 $(50 \%),{ }^{25}$ various other targets are expressed on PDAC including integrin $\alpha v \beta 6$, EGFR, and uPAR. ${ }^{10}$ Once results from other clinical studies using different tumorspecific agents become available, a cocktail of selected agents likely will be used in a personalized manner to increase the yield of fluorescence imaging. ${ }^{5}$ In addition, the targeting of tumor stroma could be pursued to increase sensitivity. Because PDAC is composed of abundant desmoplastic stroma located at the invasive front of the tumor, this tumor is particularly suited for stroma targeting. ${ }^{26}$ 
A

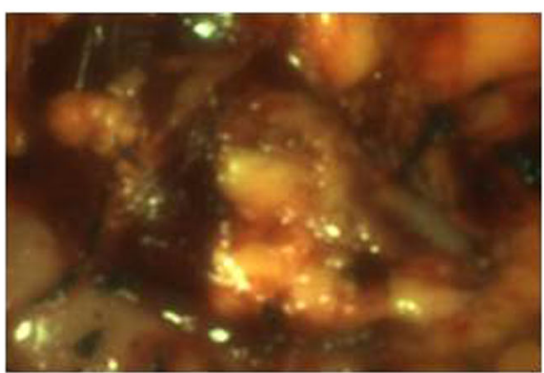

Fluorescence

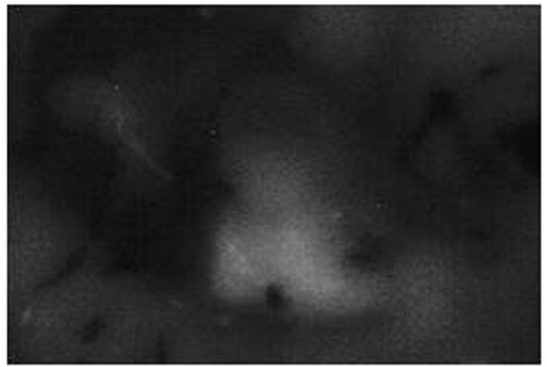

Overlay

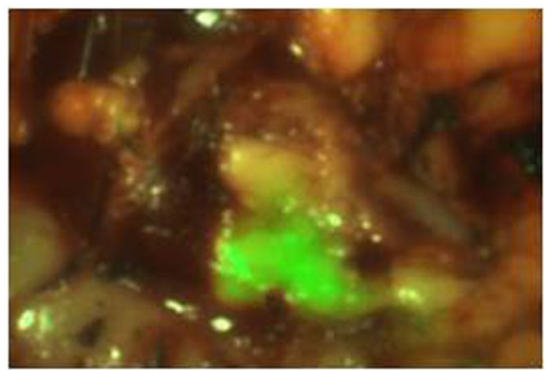

B

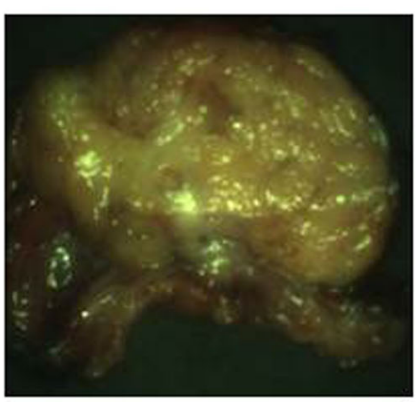

Fluorescence

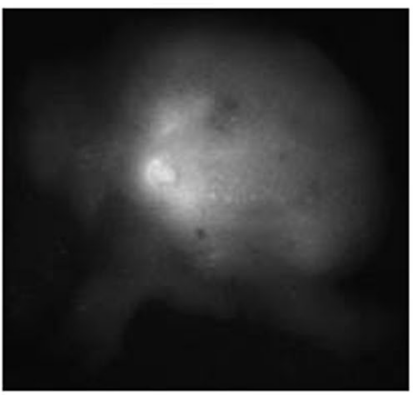

Overlay

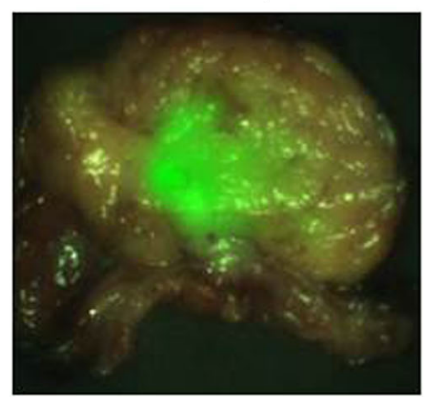

Pearl

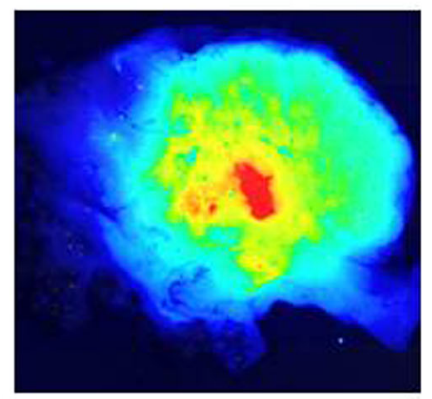

\section{Fluorescence}

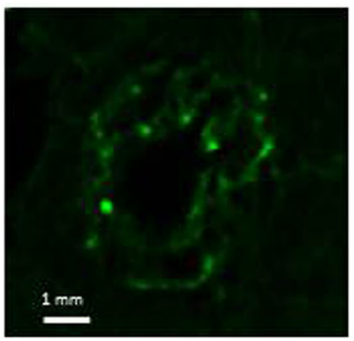

CEA

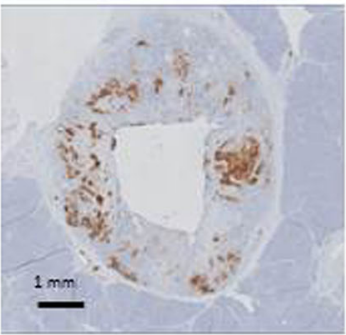

$H \& E$

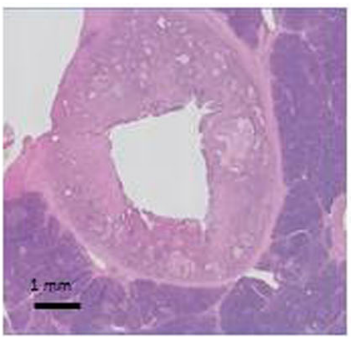

showing accumulation of SGM-101 in tumor cells. The fluorescence pattern is consistent with carcinoembryonic antigen (CEA) expression measured using immunohistochemistry (IHC, middle column), which corresponds to the site containing tumor cells visible on hematoxylin and eosin (H\&E) staining (right column). Note: The acuity of the images is suboptimal compared with the intraoperative setting because these tagged image file format (TIFF) images ( 8 bits) were subtracted from the intraoperative videos column). c Histopathologic evaluation and fluorescence signal in a primary pancreatic tumor. Fluorescence microscopy (left column)

By demonstrating that both primary tumors and small metastases can be visualized using intraoperative fluorescence imaging, this study provided the first step toward implementing fluorescence-guided PDAC surgery. However, larger clinical studies are needed to assess whether this technique allows evaluation of resectability and margin assessment (including vascular involvement) and whether this will ultimately translate into improved overall survival.

Moreover, several challenges need to be addressed before fluorescence imaging can be implemented in a broader surgical practice. Funding and awareness are required to initiate phase 3 multicenter trials. Regulatory hurdles for approval of both imaging agents and imaging

systems need to be overcome, and standardization of imaging systems is required to ensure accurate and reproducible results. $^{27}$

To the best of our knowledge, this study is the first to describe a tumor-specific imaging agent for intraoperative fluorescence imaging of PDAC. The use of a fluorescentlabeled anti-CEA antibody was safe and feasible for the detection of both primary tumors and metastases. These results underscore the great potential of image-guided surgery, but more prospective research is necessary to establish the effect on clinical decision making and overall survival. 
A

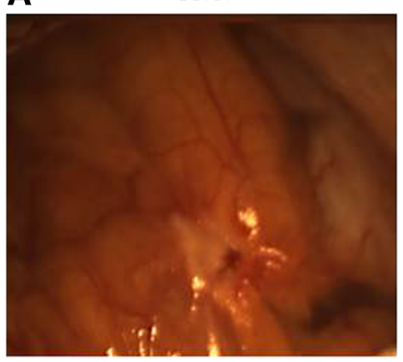

B

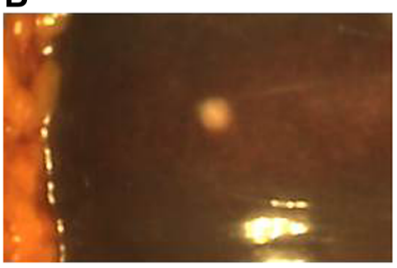

Fluorescence
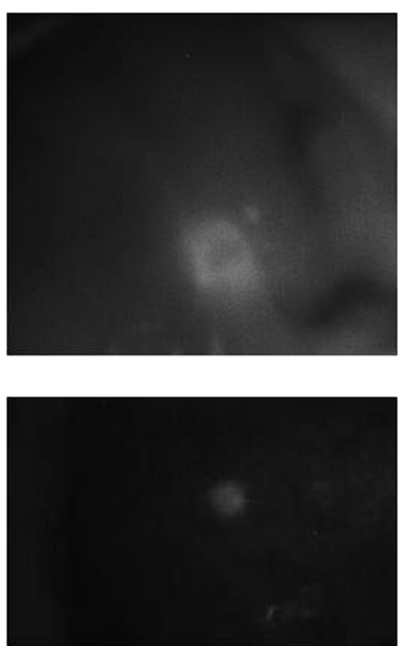

FIG. 3 Fluorescence detection of a peritoneal and liver metastasis of a pancreatic tumor. a Color (left column), fluorescence (middle left column), and merged (middle right column) images of intraoperative imaging showing a peritoneal metastasis of a pancreatic tumor and images of ex vivo imaging showing a slice from the same metastasis (right column) using the Quest imaging system. b Color (left column), fluorescence (middle left column), and merged (middle right column)

ACKNOWLEDGMENT The authors thank all the patients who participated in the study. They also thank Dr. Maxime van der Valk, Dr. Kim de Valk, Dr. Hein Handgraaf, and the CHDR nurses for their assistance and Marieke Prevoo and Shadvhi Bhairosingh for assistance with the IHC and fluorescence microscopy.

CONFLICT OF INTEREST SurgiMab (Montpellier, France) owns the SGM-101 conjugate and financed this study.

OPEN ACCESS This article is distributed under the terms of the Creative Commons Attribution 4.0 International License (http://crea tivecommons.org/licenses/by/4.0/), which permits unrestricted use, distribution, and reproduction in any medium, provided you give appropriate credit to the original author(s) and the source, provide a link to the Creative Commons license, and indicate if changes were made.

\section{REFERENCES}

1. Merkow RP, Bilimoria KY, Bentrem DJ, et al. National assessment of margin status as a quality indicator after pancreatic cancer surgery. Ann Surg Oncol. 2014;21:1067-74.

2. Chang DK, Johns AL, Merrett ND, et al. Margin clearance and outcome in resected pancreatic cancer. J Clin Oncol. 2009;27:2855-62.

3. Esposito I, Kleeff J, Bergmann F, et al. Most pancreatic cancer resections are R1 resections. Ann Surg Oncol. 2008;15:1651-60.

4. Garcea G, Dennison AR, Pattenden CJ, Neal CP, Sutton CD, Berry DP. Survival following curative resection for pancreatic ductal adenocarcinoma: a systematic review of the literature. JOP. 2008;9:99-132.

5. Vahrmeijer AL, Hutteman M, van der Vorst JR, van de Velde CJ, Frangioni JV. Image-guided cancer surgery using near-infrared fluorescence. Nat Rev Clin Oncol. 2013;10:507-18.
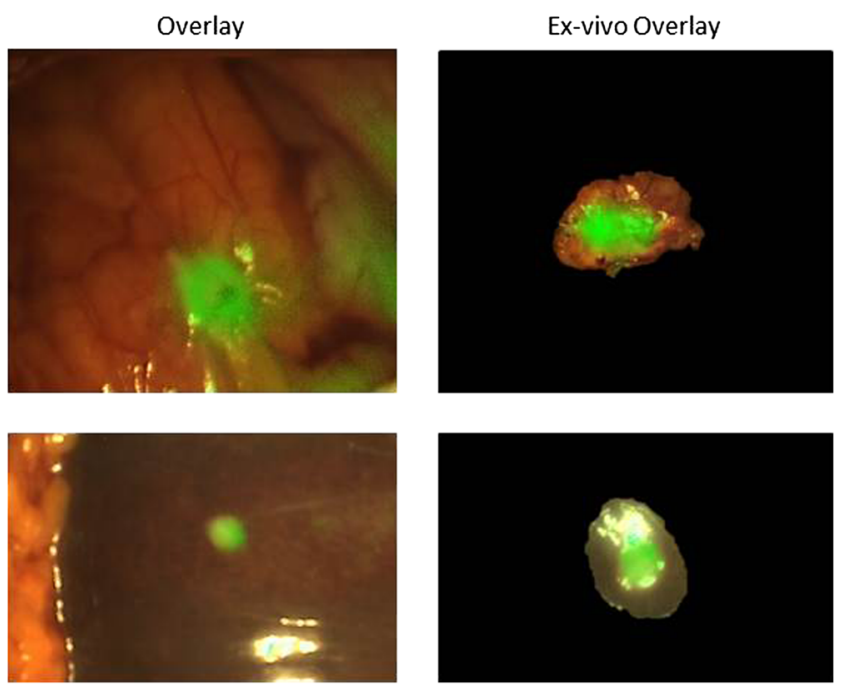

images of intraoperative imaging showing a liver metastasis of a pancreatic tumor and images of ex vivo imaging showing a slice from the same metastasis (right column) using the Quest imaging system. Note: The acuity of the images is suboptimal compared with the intraoperative setting because these tagged image file format (TIFF) images ( 8 bits) were subtracted from the intraoperative videos

6. Hutteman M, van der Vorst JR, Mieog JS, et al. Near-infrared fluorescence imaging in patients undergoing pancreaticoduodenectomy. Eur Surg Res. 2011;47:90-7.

7. Rosenthal EL, Warram JM, de Boer E, et al. Safety and tumor specificity of cetuximab-IRDye800 for surgical navigation in head and neck cancer. Clin Cancer Res. 2015;21:3658-66.

8. Stummer W, Pichlmeier U, Meinel T, et al. Fluorescence-guided surgery with 5-aminolevulinic acid for resection of malignant glioma: a randomised controlled multicentre phase III trial. Lancet Oncol. 2006;7:392-401.

9. Hoogstins CE, Tummers QR, Gaarenstroom KN, et al. A novel tumor-specific agent for intraoperative near-infrared fluorescence imaging: a translational study in healthy volunteers and patients with ovarian cancer. Clin Cancer Res. 2016;22:2929-38.

10. de Geus SW, Boogerd LS, Swijnenburg RJ, et al. Selecting tumor-specific molecular targets in pancreatic adenocarcinoma: paving the way for image-guided pancreatic surgery. Mol Imaging Biol. 2016;18:807-19.

11. Nap M, Mollgard K, Burtin P, Fleuren GJ. Immunohistochemistry of carcino-embryonic antigen in the embryo, fetus and adult. Tumour Biol. 1988;9:145-53.

12. Hammarstrom S. The carcinoembryonic antigen (CEA) family: structures, suggested functions, and expression in normal and malignant tissues. Semin Cancer Biol. 1999;9:67-81.

13. Bacac M, Fauti T, Sam J, et al. A novel carcinoembryonic antigen T-cell bispecific antibody (CEA TCB) for the treatment of solid tumors. Clin Cancer Res. 2016;22:3286-97.

14. Sahlmann CO, Homayounfar K, Niessner M, et al. Repeated adjuvant anti-CEA radioimmunotherapy after resection of colorectal liver metastases: safety, feasibility, and long-term efficacy results of a prospective phase 2 study. Cancer. 2017;123:638-49.

15. Duggan MC, Jochems C, Donahue RN, et al. A phase I study of recombinant (r) vaccinia-CEA(6D)-TRICOM and rFowlpoxCEA(6D)-TRICOM vaccines with GM-CSF and IFN-alpha-2b in patients with CEA-expressing carcinomas. Cancer Immunol Immunother. 2016;65:1353-64. 
16. Bodet-Milin C, Ferrer L, Rauscher A, et al. Pharmacokinetics and dosimetry studies for optimization of pretargeted radioimmunotherapy in CEA-expressing advanced lung cancer patients. Front Med Lausanne. 2015;2:84.

17. Schoffelen R, Boerman OC, Goldenberg DM, et al. Development of an imaging-guided CEA-pretargeted radionuclide treatment of advanced colorectal cancer: first clinical results. $\mathrm{Br} \mathrm{J}$ Cancer. 2013;109:934-42.

18. Gutowski M, Framery B, Boonstra MC, et al. SGM-101: an innovative near-infrared dye-antibody conjugate that targets CEA for fluorescence-guided surgery. Surg Oncol. 2017;26:153-62.

19. Metildi CA, Kaushal S, Pu M, et al. Fluorescence-guided surgery with a fluorophore-conjugated antibody to carcinoembryonic antigen (CEA) that highlights the tumor, improves surgical resection, and increases survival in orthotopic mouse models of human pancreatic cancer. Ann Surg Oncol. 2014;21:1405-11.

20. Kaushal S, McElroy M, Luiken GA, et al. Fluorophore-conjugated anti-CEA antibody for the intraoperative imaging of pancreatic and colorectal cancer. $J$ Gastrointest Surg. 2008;12:1938-50.
21. van Driel PB, van de Giessen M, Boonstra MC, et al. Characterization and evaluation of the artemis camera for fluorescenceguided cancer surgery. Mol Imaging Biol. 2015;17:413-23.

22. Frangioni JV. New technologies for human cancer imaging. $J$ Clin Oncol. 2008;26:4012-21.

23. Chance B. Near-infrared images using continuous, phase-modulated, and pulsed light with quantitation of blood and blood oxygenation. Ann N Y Acad Sci. 1998;838:29-45.

24. Hekman MC, Boerman OC, de Weijert M, et al. Targeted dualmodality imaging in renal cell carcinoma: an ex vivo kidney perfusion study. Clin Cancer Res. 2016;22:4634-42.

25. Chiorean EG, Coveler AL. Pancreatic cancer: optimizing treatment options, new, and emerging targeted therapies. Drug Des Devel Ther. 2015;9:29-3545.

26. Boonstra MC, Prakash J, Van De Velde CJ, et al. Stromal targets for fluorescent-guided oncologic surgery. Front Oncol. 2015;5:254.

27. Tipirneni KE, Warram JM, Moore LS, et al. Oncologic procedures amenable to fluorescence-guided surgery. Ann Surg. 2017;266:36-47. 